\title{
Estratégias de seleção de progênies de Eucalyptus saligna para produção de sementes melhoradas
}

\author{
Selection strategies in Eucalyptus saligna progenies \\ for the production of improved seeds
}

\author{
Izabele Domingues Soares ${ }^{1}$, Antonio Rioyei Higa ${ }^{2}$, Paulo César Flores Junior ${ }^{3}$, \\ Mara Luana Engel ${ }^{4}$ e Gisela Pedrassani Andrejow ${ }^{4}$
}

\section{Resumo}

O uso de técnicas de avaliação genética é fundamental para a predição de valores genéticos aditivos e genotípicos de indivíduos com potencial para seleção. O trabalho teve como objetivo propor estratégias de seleção de progênies para composição de Pomar de Sementes por Mudas - PSM a partir da avaliação genética e do efeito da interação genótipos $\mathrm{x}$ ambientes considerando a produção de madeira de Eucalyptus saligna. Testes de progênies foram instalados em três municípios do estado de Santa Catarina (Três Barras, Mafra e Canoinhas), Brasil, em delineamento experimental de blocos casualizados, com 50 tratamentos (progênies), cinco blocos e seis plantas por parcela. As estimativas dos parâmetros genéticos foram realizadas para a variável diâmetro à altura do peito e obtidas utilizando o programa genético-estatístico SELEGEN-REML/BLUP, no qual, foram utilizados o modelo 1 para avaliação individual dos testes de progênies e o modelo 4 para a análise conjunta. Para análise de produtividade, estabilidade e adaptabilidade, utilizou-se o método MHPRVG. Foram encontrados valores que indicam um expressivo controle genético e altas acurácias $(>0,651)$ na seleção entre progênies. Na análise conjunta dos locais a correlação genética entre o comportamento das progênies foi baixa $(0,322)$, sugerindo que uma população de melhoramento única não seria vantajosa utilizando esse método. Pelo método MHPRVG, a correlação genética encontrada $(0,79)$ conduz à seleção dos melhores genótipos para composição de um único PSM para os três municípios onde foram instalados os ensaios e na seleção por local, Três Barras apresentou as progênies de maior ganho genético (> 8,54\%) comparada com Mafra e Canoinhas (> 4,39\%; 5,77\%). Para possível comercialização de sementes é recomendado a seleção de três indivíduos por família pelo método MHPRVG para composição de um único PSM para os três municípios; a curto prazo, visando a obtenção de maiores ganhos genéticos mediante o uso de sementes melhoradas, recomenda-se a seleção de dois indivíduos por família para compor um único PSM para suprir Três Barras (seleção por local) e um segundo PSM formado pelo método MHPRVG com seleção de três indivíduos por família para suprir Mafra e Canoinhas.

Palavras-chave: Pomar de Sementes por Mudas; parâmetros genéticos; REML/BLUP.

\begin{abstract}
The use of techniques of genetic evaluation is essential for the prediction of additive and genotypic values of individuals with potential for selection. The aim of this work is proposing selection strategies in progenies of Eucalyptus saligna for composition of a Seedling Seed Orchard - PSM based on the genetic evaluation and the effect of genotypes vs. environments interaction for wood production. The progeny tests were established in three municipalities in the state of Santa Catarina (Três Barras, Mafra and Canoinhas), Brazil, under an experimental design of randomized blocks, with 50 entries (progenies), five blocks and six plants per plot. The estimates of the genetic parameters were performed for the variable diameter at breast height and obtained using the genetic-statistical program SELEGEN-REML / BLUP, in which we used the model 1 for the assessment of each test individually and the Model 4 for the joint analysis. For yield, stability and adaptability, we used the method MHPRVG. We found values that indicate a significant genetic control and high accuracies $(>0.651$ ) when the selection was carried out among the progenies. In the joint analysis the genetic correlation for the performance of the progenies was low (0.322), suggesting that a
\end{abstract}

\footnotetext{
${ }^{1}$ Doutoranda em Engenharia Florestal. UFPR - Universidade Federal do Paraná. Av. Lothário Meissner, 3400 - Jardim Botânico - 80210170 - Curitiba, PR, Brasil. E-mail: izabele.soares@gmail.com

2Professor Titular no Departamento de Engenheiro Florestal. UFPR - Universidade Federal do Paraná. Av. Lothário Meissner, 3400 - Jardim Botânico - 80210170 - Curitiba, PR, Brasil. E-mail: antonio.higa@gmail.com

3Professor Substituto no Departamento de Engenheiro Florestal. UFPR - Universidade Federal do Paraná. Av. Lothário Meissner, 3400 - Jardim Botânico - 80210170 - Curitiba, PR, Brasil. E-mail: paulocesarfloresjunior@gmail.com

${ }^{4}$ Pesquisador Mestre em Engenharia Florestal. MWV Rigesa / Divisão Florestal - Três Barras. Av. Rigesa, 2929 - Caixa-postal: 31- 89490000 - Três Barras, SC, Brasil. E-mail: maraluana_pzo@hotmail.com; gisela.andrejow@mwv.com
} 
single population of improvement would not be advantageous. In turn, by using the MHPRVG method, the genetic correlation found (0.79) leads to the selection of the best genotypes for composition of a single PSM for the three municipalities. In selection by location, Três Barras presented the progenies of greater genetic gain (> 8,54\%) compared to Mafra and Canoinhas (> 4.39\%; 5.77\%). For an eventual commercialization of the seeds, the suggestion is the selection of three individuals per family by the MHPRVG method for composition of a single PSM for the three municipalities; in short term, focusing obtaining larger genetic gains through the use of improved seeds, selection of two individuals per family to compose a single PSM in Três Barras (selection by site) is recommended. Another PSM formed by MHPRVG method with selection of three individuals per family is recommended to supply the demands of improved seeds coming from Mafra and Canoinhas.

Keywords: Seedling seed orchard; Genetic parameter; REML/BLUP.

\section{INTRODUÇÃO}

O sucesso global da indústria brasileira de base florestal é resultado da alta produtividade das árvores plantadas. Em 2014, a produtividade média dos plantios brasileiros de eucalipto atingiu 39 $\mathrm{m}^{3}$ /ha/ano (IBÁ, 2015) e dentre as principais espécies desse gênero cultivadas no Brasil encontra-se o Eucalyptus saligna, considerada uma das espécies mais versáteis e indicadas para usos múltiplos. De acordo com Barros et al. (1990), a espécie tolera solos moderadamente férteis, com boa retenção de umidade, porém devem ser bem drenados. As características da madeira a tornam indicada para laminação, móveis, estruturas, caixotaria, postes, celulose e carvão (FERREIRA, 1979).

$\mathrm{O}$ aumento e a manutenção da produtividade das plantações florestais são alcançados, principalmente, por meio do melhoramento genético. Um programa de melhoramento, dependendo da espécie, pode contemplar tanto procedimento sexuado (sementes) como assexuado (clones). Um dos aspectos importantes no uso de sementes para plantios comerciais é a garantia da manutenção de ampla base genética, ou seja, maior variabilidade entre os indivíduos, possibilitando a obtenção de materiais distintos e adaptados a diferentes condições de solo e clima (ANGELI, 2006).

São diversas as formas de obtenção de sementes melhoradas para uso comercial. Dentre estas tem-se o estabelecimento de Pomares de Sementes por Mudas - PSM. O PSM é implementado a partir da seleção entre e dentro de famílias em um teste de progênies após sucessivos desbastes. Nesta modalidade de produção de sementes melhoradas tem-se o controle da genealogia de cada árvore selecionada, possibilitando a obtenção de estimativas de parâmetros genéticos e predição de ganho com seleção (PIRES et al., 2011). A intensidade de seleção tanto entre como dentro das famílias é função dos objetivos do melhorista, do número de famílias, do número de plantas por parcela, ou mesmo do ganho genético desejado (PIRES et al., 2011).

O uso de técnicas de avaliação genética, com base em modelos mistos do tipo REML/BLUP (máxima verossimilhança restrita/melhor predição linear não viciada) é fundamental para a predição de valores genéticos aditivos e genotípicos de indivíduos com potencial para seleção, tanto em nível intrapopulacional como interpopulacional (RESENDE; DIAS, 2000). Sendo assim, esse procedimento vem sendo aplicado com sucesso no melhoramento de espécies perenes no Brasil, principalmente para espécies do gênero Eucalyptus (RESENDE et al., 1993) e Pinus (RESENDE et al., 1996).

Contudo, deve-se salientar que o desempenho de genótipos em relação ao ambiente requer especial atenção, devido à sua interferência nos processos de seleção. Entre as alternativas para se amenizar a influência da interação genótipos x ambientes, tem sido recomendado o emprego de cultivares com ampla adaptabilidade e boa estabilidade (BERTOLUCCI et al., 1995; ROSADO et al., 2012). A possibilidade de avaliar um teste de progênies instalado em vários locais reduz o efeito dessa interação no resultado da seleção, o que permite a utilização mais ampla do material selecionado (BUENO et al., 2001).

Assim, o objetivo do trabalho foi propor estratégias de seleção de progênies para composição de PSM a partir da avaliação genética, considerando a produção de madeira de Eucalyptus saligna, em nível de locais e para as regiões de abrangência dos experimentos estudados, além da interação genótipos $\mathrm{x}$ ambientes por meio da adaptabilidade e estabilidade das progênies. 


\section{MATERIAL E MÉTODOS}

Os testes de progênies de polinização aberta de E. saligna foram instalados em três municípios no estado de Santa Catarina: Três Barras, Mafra e Canoinhas, nos quais as características edafoclimáticas estão apresentadas na Tabela 1.

Tabela 1. Características edafoclimáticas das três regiões de estudo.

Table 1. Soil and climate characteristics of the three study areas.

\begin{tabular}{lccccl}
\hline LOCAL & LAT. & LONG. & CLIMA & ALT. & TIPO DE SOLO \\
\hline Três Barras & $26^{\circ} 04^{\prime} \mathrm{N}$ & $50^{\circ} 15^{\prime} \mathrm{E}$ & Cfb & 795 & Latossolo Bruno/Vermelho-Escuro Álico A húmico \\
Mafra & $26^{\circ} 04^{\prime} \mathrm{N}$ & $50^{\circ} 09^{\prime} \mathrm{E}$ & Cfb & 800 & Cambissolo Álico Tb A moderado \\
Canoinhas & $26^{\circ} 05^{\prime} \mathrm{N}$ & $50^{\circ} 03^{\prime} \mathrm{E}$ & Cfb & 815 & Cambissolo Álico Tb A proeminente \\
\hline
\end{tabular}

Lat.- latitude; Long.- longitude; Alt.- altitude em metros.

Os mesmos foram plantados em janeiro de 2006, em delineamento experimental de blocos casualizados, com 50 tratamentos (progênies), cinco blocos, seis plantas por parcela e espaçamento de 2,5 m x 3,0 m. Em todos os testes foram avaliados o diâmetro à altura do peito (DAP) e a altura total da árvore $(\mathrm{H})$, mensurados aos 5 anos de idade. A partir das análises de correlação genética realizadas entre as duas variáveis, optou-se pela utilização da variável DAP para a seleção, em virtude da sua fácil mensuração, uma vez que o volume é calculado diretamente dessas variáveis e, por conseguinte, dependente delas.

A seleção das melhores progênies para composição de PSM foi realizada a partir das estimativas dos parâmetros genéticos para a variável DAP, sendo obtidas pelo programa genético-estatístico SELEGEN-REML/BLUP, desenvolvido por Resende (2002). A avaliação individual dos testes de progênies foi efetuada pelo modelo 1, metodologia do modelo linear misto (aditivo univariado) REML/BLUP, assumindo progênies como sendo de meios irmãos: $\gamma=X r+Z a+W p+e$; em que: $\mathrm{y}=$ vetor de dados; $r$ = vetor dos efeitos de repetição (assumidos como fixos) somados à média geral; a = vetor dos efeitos genéticos aditivos individuais (assumidos como aleatórios); $\mathrm{p}=$ vetor dos efeitos de parcela; $\mathrm{e}=$ vetor de erros ou resíduos (aleatórios); $X, Z, W=$ matrizes de incidência para os referidos efeitos. A análise conjunta dos testes de progênies envolvendo os três locais simultaneamente foi realizada com base no seguinte modelo (modelo 4): $y=X r+Z g+W p+T i+e$, sendo semelhante ao primeiro modelo, com a inclusão do vetor $i$, que se refere ao efeitos da interação genótipos $\mathrm{x}$ ambientes (aleatório) da matriz de incidência $T$, que está associada a esse efeito.

A seleção de progênies considerando simultaneamente a produtividade, adaptabilidade e estabilidade foi realizada pelo método da média harmônica da performance relativa dos valores genéticos (MHPRVG) (modelo 51), empregando-se também o programa SELEGEN - REML/BLUP. Para esta análise foi utilizado o modelo estatístico representado pela expressão: $y=X r+Z g+W p+T i+e$ (RESENDE, 2007), em que: $y, r, g$, $i$ e $e$ são os vetores de dados referentes aos efeitos de repetição (fixos), genotípico (aleatórios), parcelas (aleatórios), da interação G x A (aleatórios) e do resíduo (aleatório), respectivamente, $X, Z W$ e T representam as matrizes de incidência para os referidos efeitos.

Para as estratégias de melhoramento via sexuada (sementes) foram utilizados valores genéticos aditivos (RESENDE, 2002). As estimativas de ganhos genéticos foram baseadas na seleção de indivíduos com valores positivos para os efeitos genéticos aditivos da variável DAP. Estas estimativas foram feitas na seleção por local, na seleção para os três locais simultaneamente e na seleção considerando os atributos produtividade, estabilidade e adaptabilidade. Para isso, foi utilizada a seguinte fórmula: $\mathrm{GS}=\bar{X}_{\mathrm{a}} / \bar{X}_{0} * 100$, em que $\bar{X}_{\mathrm{a}}$ é a média dos valores genéticos aditivos das progênies selecionadas; e $\bar{X}_{0}$ é a média geral dada pelo Selegen. Para avaliar o tamanho efetivo populacional (Ne) das progênies foi utilizada a expressão de acordo com Resende e Bertolucci (1995):

$$
N e=\frac{4 N_{f} \bar{K}_{f}}{K_{f}+3+\left(\sigma_{K f}^{2} / K_{f}^{2}\right)}
$$

Em que: $\bar{K}_{f}$ é o número médio de indivíduos selecionados por progênie; $\sigma_{K f}^{2}$ é a variância do número de indivíduos selecionados por progênie; e $N_{f}$ é o número de progênies selecionadas. 
Soares et al. - Estratégias de seleção de progênies de

Eucalyptus saligna para produção de sementes melhoradas

\section{RESULTADOS E DISCUSSÃO}

As estimativas de correlações genéticas entre DAP x $\mathrm{H}$ apresentaram os seguintes valores respectivamente: 0,84 e 0,89 . Isso demonstra associações positivas e consideravelmente altas, o que favorece a seleção baseada em apenas uma variável, no caso DAP por ser de fácil mensuração, pois automaticamente levará ao melhoramento indireto da variável altura. A alta associação genética entre os caracteres de crescimento para as espécies florestais é citada por diversos autores como Kageyama (1980), Sampaio (1996) e Sturion (1993).

A precisão experimental avaliada pelos coeficientes de variação experimental (CVe) foi boa, com valores variando de $13,54 \%$ a $15,78 \%$. Os parâmetros genéticos, os componentes da variância e os coeficientes de variação das progênies do teste de progênies de E. saligna, determinados para esta variável em cada local, estão apresentados na Tabela 2.

Os valores de variância genética aditiva individual e variância entre parcelas para Três Barras, foram maiores em relação aos outros dois locais. Isto de certa forma refletiu nos valores estimados da herdabilidade individual no sentido restrito (0,20 para Três Barras e 0,11 para Mafra e Canoinhas) ajustadas para os efeitos de parcela. Para a herdabilidade aditiva individual, Resende e Higa (1994) consideram de 0,01 a 0,15 como baixas; de 0,15 a 0,50 são medianas; e acima de 0,50 altas. Baseado nisso, a herdabilidade aditiva das progênies em Três Barras apresentaram valor mediano $(0,20)$ e, para os demais locais, valor de baixa magnitude $(0,11$ para ambos) representando elevado fator ambiental. A herdabilidade média de progênies (0,57 para Três Barras; 0,42 para Mafra e 0,45 para Canoinhas) pode ser considerada alta para a variável DAP e isso permite inferir que há boa chance de sucesso na seleção entre progênies para essa variável, possibilitando ganhos genéticos significativos, além das altas acurácias observadas $(0,76,0,67$ e 0,65).

De modo geral, os valores apresentaram-se semelhantes para os locais, para a variável DAP avaliada e, devido às variâncias observadas, ganhos genéticos expressivos poderão ser obtidos pela seleção por local.

Tabela 2. Estimativas de parâmetros genéticos para o caráter diâmetro à altura do peito (DAP) em testes de progênies de Eucalyptus saligna nos três locais de plantio e pela análise conjunta, avaliados aos 5 anos de idade.

Table 2. Estimates of genetic parameters for diameter at breast height (DBH) in progeny tests of Eucalyptus saligna per location and by joint analysis, evaluated at 5 years of age.

\begin{tabular}{|c|c|c|c|c|}
\hline \multirow{2}{*}{ PARÂMETRO } & \multicolumn{3}{|c|}{ LOCAL } & \multirow{2}{*}{ ANÁLISE CONJUNTA } \\
\hline & Três Barras & Mafra & Canoinhas & \\
\hline$\overline{V_{a}}$ & 4,17 & 2,61 & 3,34 & 1,75 \\
\hline$V_{\text {parc }}$ & 0,66 & 0,58 & 0,23 & 0,084 \\
\hline$V_{\text {int }}$ & - & - & - & 0,92 \\
\hline$V_{e}$ & 16,55 & 21,15 & 27,13 & 23,22 \\
\hline$V_{f}$ & 21,38 & 24,35 & 30,71 & 25,97 \\
\hline$h_{a}^{2}$ & $0,20+-0,068$ & $0,11+-0,06$ & $0,11+-0,05$ & $0,07+-0,024$ \\
\hline$h_{a j}^{2}$ & 0,20 & 0,11 & 0,11 & - \\
\hline$c_{\text {parc }}^{2}$ & 0,031 & 0,024 & 0,008 & 0,003 \\
\hline$c^{2}{ }_{i n t}$ & - & - & - & 0,035 \\
\hline$h_{m p}^{2}$ & 0,57 & 0,42 & 0,45 & 0,28 \\
\hline$A c_{\text {prog }}$ & 0,755 & 0,651 & 0,669 & 0,526 \\
\hline$h_{a d}^{2}$ & 0,16 & 0,09 & 0,09 & 0,05 \\
\hline$r g_{\text {loc }}$ & - & - & - & 0,32 \\
\hline$C V_{g i}^{o} \%$ & 14,57 & 10,40 & 12,69 & - \\
\hline$C V_{g p}^{s *} \%$ & 7,28 & 5,20 & 6,34 & - \\
\hline$C V_{e}^{g p} \%$ & 14,15 & 13,54 & 15,78 & - \\
\hline$C V_{r}$ & 0,52 & 0,38 & 0,40 & - \\
\hline PEV & 0,45 & 0,38 & 0,46 & 0,32 \\
\hline SEP & 0,67 & 0,61 & 0,68 & 0,56 \\
\hline Média geral & 14,02 & 15,55 & 14,41 & 14,61 \\
\hline \multicolumn{5}{|c|}{ 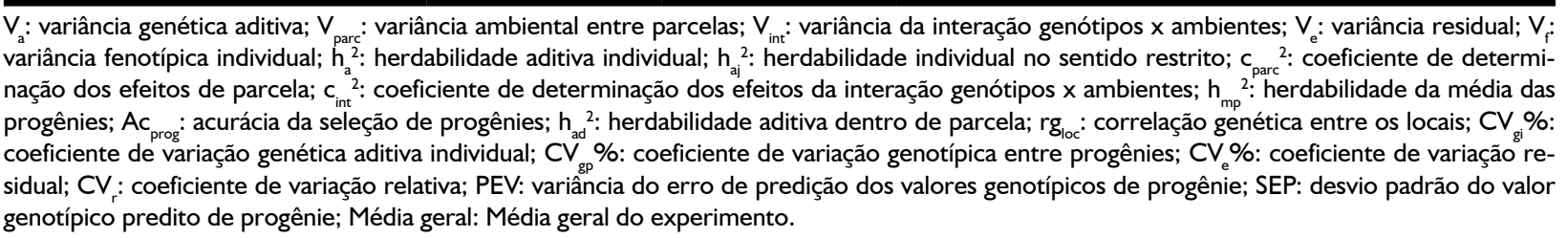 } \\
\hline
\end{tabular}


A correlação genética entre o comportamento das progênies considerando os três locais foi baixa $(0,32)$ para a variável DAP. Segundo Resende (2002), uma população de melhoramento única, com seleção de materiais estáveis (seleção pela média de locais), deve ser adotada quando o valor da correlação genética entre locais estiver compreendido entre os valores 0,70 e 0,90.

Em relação ao ordenamento das melhores progênies baseadas na produtividade, adaptabilidade e estabilidade em Três Barras, Mafra e Canoinhas pelo método MHPRVG, a progênie C120 apresentou a melhor produtividade, estabilidade e adaptabilidade de acordo com os resultados da seleção, indicando que, independentemente do ambiente, essa progênie tem bom desempenho. Esse mesmo padrão também foi observado para a maioria das progênies selecionadas (Tabela 3), o que indica que o critério de seleção simultânea (com dados de adaptabilidade, estabilidade e produção) é eficaz para selecionar as melhores progênies pelo seu valor genotípico (produção). Ao analisarem a seleção simultânea de clones de eucalipto de acordo com a produtividade, estabilidade e adaptabilidade, Rosado et al. (2012) concluíram que o método MHPRVG proporciona seleção otimizada e deve fazer parte dos critérios seletivos de clones de eucalipto para construir populações de melhoramento.

Tabela 3. Ordenamento das progênies do Teste de Progênies de Eucalyptus saligna resultantes da análise de estabilidade e adaptabilidade (MHPRVG e MHPRVG*MG) entre os três locais de estudo.

Table 3. Progenies ordering of Eucalyptus saligna resulting from analysis of stability and adaptability (MHPRVG and MHPRVG*MG) among the three locations of study.

\begin{tabular}{|c|c|c|c|}
\hline ORDEM & GENÓTIPO & MHPRVG & MHPRVG*MG \\
\hline 1 & C120 & 1,0847 & 15,8907 \\
\hline 2 & C157 & 1,0762 & 15,7667 \\
\hline 3 & C138 & 1,0725 & 15,7131 \\
\hline 4 & C136 & 1,0625 & 15,5654 \\
\hline 5 & C162 & 1,0604 & 15,5354 \\
\hline 6 & $\mathrm{C} 80$ & 1,0593 & 15,5187 \\
\hline 7 & C153 & 1,0518 & 15,4091 \\
\hline 8 & C58 & 1,0479 & 15,3528 \\
\hline 9 & $\mathrm{C} 82$ & 1,0439 & 15,2939 \\
\hline 10 & C34 & 1,0433 & 15,2847 \\
\hline 11 & C36 & 1,0426 & 15,2748 \\
\hline 12 & C78 & 1,0406 & 15,2450 \\
\hline 13 & C102 & 1,0342 & 15,1521 \\
\hline 14 & C168 & 1,0337 & 15,1446 \\
\hline 15 & $\mathrm{C} 84$ & 1,0283 & 15,0645 \\
\hline 16 & C149 & 1,0253 & 15,0216 \\
\hline 17 & C118 & 1,0238 & 14,9985 \\
\hline 18 & C160 & 1,0192 & 14,9322 \\
\hline 19 & C169 & 1,0184 & 14,9203 \\
\hline 20 & C119 & 1,0156 & 14,8795 \\
\hline 21 & C77 & 1,0133 & 14,8454 \\
\hline 22 & C103 & 1,0093 & 14,7874 \\
\hline 23 & C163 & 1,0073 & 14,7577 \\
\hline 24 & C114 & 1,0067 & 14,7493 \\
\hline 25 & C142 & 1,0051 & 14,7257 \\
\hline 26 & C108 & 1,0046 & 14,7173 \\
\hline 27 & $\mathrm{C} 83$ & 1,0036 & 14,7036 \\
\hline 28 & C171 & 1,0005 & 14,6576 \\
\hline 29 & C75 & 1,0000 & 14,6505 \\
\hline
\end{tabular}

Foi visto que $100 \%$ das progênies selecionadas pelo método MHPRVG situam-se entre as melhores progênies na análise conjunta. Sendo assim, os cálculos de ganho genético e Ne considerando a seleção pelo método, foram baseados nos valores aditivos de cada progênie ranqueada e também encontradas na análise conjunta. A correlação genética entre o comportamento das progênies pelo método da média harmônica foi de 0,79 e conduz à seleção dos melhores genótipos para ambos os locais. Neste caso, recomenda-se um Pomar de Sementes por Mudas único para Três Barras, Mafra e Canoinhas. Conforme resultados apresentados na Tabela 4 é possível considerar os atributos produtividade, adaptabilidade e estabilidade como uma das estratégias para seleção de progênies para composição de PSM. 
Tabela 4. Tamanho efetivo populacional ( $\mathrm{Ne}$ ), ganho genético predito, média dos valores genéticos aditivos (a), média geral e nova média da população selecionada para as diferentes estratégias de seleção de progênies de Eucalyptus saligna, nos cinco blocos.

Table 4. Effective population size $(\mathrm{Ne})$, predicted genetic gain, means of genetic additive values (a), general mean and new mean of the population for the different selection strategies in progenies of Eucalyptus saligna.

SELEÇÃO DE TRÊS INDIVÍDUOS POR FAMÍLIA

\begin{tabular}{lccccc}
\hline \multirow{2}{*}{ ESTIMATIVAS } & \multicolumn{3}{c}{ Seleção por Local } & $\begin{array}{c}\text { Seleção pela Análise } \\
\text { Conjunta }\end{array}$ & $\begin{array}{c}\text { Seleção pelo Método } \\
\text { MHPRVG }\end{array}$ \\
\cline { 2 - 4 } & Três Barras & Mafra & Canoinhas & 87,55 & 58,00 \\
Ne & 90,20 & 85,99 & 88,20 & 3,943 & 5,108 \\
Manho & 8,545 & 4,396 & 5,776 & 0,576 & 0,746 \\
Média de a & 1,198 & 0,684 & 0,833 & 14,608 & 14,608 \\
Nova Média & 14,023 & 15,550 & 14,413 & 15,184 & 15,354 \\
\hline
\end{tabular}

SELEÇÃO DE DOIS INDIVÍDUOS POR FAMÍLIA

\begin{tabular}{lccccc}
\hline \multirow{2}{*}{ ESTIMATIVAS } & \multicolumn{3}{c}{ Seleção por Local } & $\begin{array}{c}\text { Seleção pela Análise } \\
\text { Conjunta }\end{array}$ & $\begin{array}{c}\text { Seleção pelo Método } \\
\text { MHPRVG }\end{array}$ \\
\cline { 2 - 4 } & Três Barras & Mafra & Canoinhas & 70,4 & 46,4 \\
Ne & 73,27 & 69,76 & 71,67 & 4,075 & 5,268 \\
Ganho & 8,951 & 4,612 & 5,979 & 0,595 & 0,770 \\
Média de a & 1,255 & 0,717 & 0,862 & 14,608 & 14,608 \\
Média Geral & 14,023 & 15,550 & 14,413 & 15,203 & 15,377 \\
Nova Média & 15,278 & 16,268 & 15,275 & &
\end{tabular}

\begin{tabular}{|c|c|c|c|c|c|}
\hline \multirow{2}{*}{ ESTIMATIVAS } & \multicolumn{3}{|c|}{ Seleção por Local } & \multirow{2}{*}{$\begin{array}{c}\text { Seleção pela Análise } \\
\text { Conjunta }\end{array}$} & \multirow{2}{*}{$\begin{array}{c}\text { Seleção pelo Método } \\
\text { MHPRVG }\end{array}$} \\
\hline & Três Barras & Mafra & Canoinhas & & \\
\hline $\mathrm{Ne}$ & 47 & 44 & 46 & 44 & 29 \\
\hline Ganho & 9,369 & 5,008 & 6,186 & 4,267 & 5,441 \\
\hline Média de a & 1,314 & 0,779 & 0,892 & 0,623 & 0,795 \\
\hline Média Geral & 14,023 & 15,550 & 14,413 & 14,608 & 14,608 \\
\hline Nova Média & 15,337 & 16,329 & 15,305 & 15,231 & 15,403 \\
\hline
\end{tabular}

É importante ressaltar que na seleção de um indivíduo por família, tamanhos efetivos são iguais ao número de progênies selecionadas. Essa relação é derivada da expressão descrita por Resende e Bertolucci (1995). Pelo método MHPRVG, a seleção de um indivíduo por família proporcionou o menor Ne (29), valor correspondente ao número de famílias selecionadas. Isso demonstra uma certa restrição na base genética, contudo, dependerá da quantidade de indivíduos selecionados por família.

Analisando adaptabilidade e estabilidade das progênies (seleção pelo método MHPRVG), a seleção de um indivíduo por família proporcionou o maior ganho genético, no entanto, o menor Ne. Esse foi um resultado generalizado, ou seja, observado na seleção por local e na seleção pela análise conjunta, além disso, é justificado pelo aumento da média do valor genético aditivo ao selecionar apenas um indivíduo por família. Namkoong (1974), citado por Oda et al. (1989), considera que a redução da população pode ocasionar riscos de endogamia, diminuindo o vigor da floresta. Por outro lado, para se ter ganhos imediatos a pressão de seleção deve ser alta (ODA et al., 1989).

Considerando que, para prevenção de depressão endogâmica um Ne de 50 é o suficiente (PIRES et al., 2011), para a seleção resultante do método MHPRVG é indicado selecionar três indivíduos por família ( $\mathrm{Ne}=58$; GS=5,11\%), pois o Ne está acima do mínimo recomendado para evitar endogamia. Para uma possível comercialização das sementes, utilizar um único PSM resultante da seleção simultânea para produtividade, adaptabilidade e produtividade para os três locais pode ser muito mais vantajoso economicamente, além de gerar indivíduos mais adaptados e estáveis.

De acordo com a Tabela 4, na seleção por local, Três Barras apresenta as progênies de maior ganho genético ao comparar-se com Mafra e Canoinhas. A seleção por local seria vantajosa para a empresa/produtor responsável, pois acarretaria em um maior ganho genético e seria uma estratégia de melhoramento a curto prazo. Uma seleção por local de dois indivíduos por família pode ser uma estratégia quando se deseja manter um Ne acima do recomendado ( $\mathrm{Ne}>50$ ). Admitindo que o ganho genético a partir da análise MHPRVG é maior que o ganho das progênies de Mafra selecionando dois indivíduos por família $(4,61 \%)$ e está praticamente equiparado com o ganho genético nas progênies de Canoinhas (5,97 \%), recomenda-se um PSM para os dois locais com três 
indivíduos por família pela seleção usando o método MHPRVG (ganho de 5,11\%) e mais um PSM para Três Barras (seleção por local) considerando uma seleção de dois indivíduos por família, alcançando um ganho genético de $8,95 \%$. Desta forma, seria viável economicamente um total de dois PSM para atender a empresa/produtor; considerando também o ganho genético; e a manutenção do Ne adequado de indivíduos.

\section{CONCLUSÕES}

É recomendada a seleção de três indivíduos por família pelo método MHPRVG para composição de um único PSM para os três locais estudados, considerando a possível comercialização das sementes.

A curto prazo, para obtenção de maiores ganhos genéticos mediante o uso de sementes melhoradas, recomenda-se a seleção de dois indivíduos por família para compor um único PSM para suprir Três Barras (seleção por local) e a seleção de três indivíduos por família para compor um segundo PSM formado pelo método MHPRVG para suprir Mafra e Canoinhas.

\section{REFERÊNCIAS BIBLIOGRÁFICAS}

ANGELI, P. H. M. S. A. Implantação e manejo de florestas comerciais. Documentos florestais IPEF, Piracicaba, n.18, 2006. 16 p.

BARROS, N. F., NOVAIS, R. F., CARDOSO, J. R.; MACEDO, P. R. O. Algumas relações solo-espécies de eucalipto em suas condições naturais. In: BARROS, N. F., NOVAIS, R. F. (Eds.) Relação solo-eucalipto. Viçosa: Folha de Viçosa, 1990. p.1-24

BERTOLUCCI, F.; REZENDE, G.; PENCHEL, R. Produção e utilização de híbridos de eucalipto. Silvicultura, São Paulo, v. 51, p. 12-16, 1995.

BUENO, L. C. S.; MENDES, A. N. G.; CARVALHO, S. P. Melhoramento genético de plantas. Lavras: Editora da UFLA, 2001.

FERREIRA, M. Escolha de Espécies de Eucalipto. Circular Técnica IPEF, Piracicaba, v.47, 1979. 30 p.

IBÁ - INDÚSTRIA BRASILEIRA DE ÁRVORES. Relatório IBÁ 2015 ano base 2014. Brasília: IBÁ, 2015. 64 p.

KAGEYAMA, P. Y. Variação genética em progênies de uma população de Eucalyptus grandis (Hill) Maiden.1980. 125 p. Tese (Doutorado) - Escola Superior de Agricultura Luiz de Queiroz, Piracicaba, 1980.

ODA, S.; MENCK, A. L. M.; VENCOVSKY, R. Problemas no melhoramento genético clássico do eucalipto em função da alta intensidade de seleção. Revista IPEF, Piracicaba, n.41/42, 1989.

PIRES, I. E.; RESENDE, M. D. V.; SILVA, R. L.; RESESENDE JUNIOR, M. F. R. Genética Florestal. Viçosa: Arka, 2011.

RESENDE, M. D. V. Matemática e estatística na análise de experimentos e no melhoramento genético. Colombo: Embrapa Florestas, 2007. 562 p.

RESENDE, M. D. V. Genética biométrica e estatística no melhoramento de plantas perenes. Brasília: Embrapa Informação Tecnológica; Colombo: Embrapa Florestas, 2002. 975 p.

RESENDE, M. D. V.; BERTOLUCCI, F. L. G. Maximization of genetic gain with restriction on effective population size and inbreeding in Eucalyptus grandis. In: IUFRO CONFERENCE "EUCALYPT PLANTATIONS: IMPROVING FIBRE YIELD AND QUALITY", 1995, Hobart. Proceedings... Hobart: CRC for Temperate Hardwood Forestry, p.167-170, 1995. 
RESENDE, M. D. V.; DIAS, L. A. S. Aplicação da metodologia de modelos mistos (REML/BLUP) na estimação de parâmetros genéticos e predição de valores genéticos em espécies frutíferas. Revista Brasileira de Fruticultura, Jaboticabal, v. 22, n. 1, p. 44-52, 2000.

RESENDE, M. D. V.; HIGA, A. R. Estimação de valores genéticos no melhoramento de Eucaliptus: seleção em um caráter com base em informações do indivíduo e seus parentes. Boletim de Pesquisa Florestal, Colombo, n. 28/29, p. 11-36, 1994.

RESENDE, M. D. V.; PRATES, D. F; YAMADA, C. K.; JESUS, A. Estimação de componentes de variância e predição de valores genéticos pelo método da máxima verossimilhança restrita (REML) e melhor predição linear não viciada (BLUP) em Pinus. Boletim de Pesquisa Florestal, Colombo, n. 32/33, p. 18-45, 1996.

RESENDE, M. D. V.; HIGA, A. R.; LAVORANTI, O. J. Predição de valores genéticos no melhoramento de Eucalyptus: melhor predição linear. In: CONGRESSO FLORESTAL BRASILEIRO, 7., 1993, Curitiba. Anais... Curitiba: SBF, 1993. p. 144-147.

ROSADO, A. M.; ROSADO, T. B.; ALVES, A. A.; LAVIOLA, B. G.; BHERING, L. L. Seleção simultânea de clones de eucalipto de acordo com produtividade, estabilidade e adaptabilidade. Pesquisa agropecuária brasileira, Brasília, v. 47, n. 7, 2012.

SAMPAIO, P. T. B. Variação genética entre procedências e progênies de Pinus oocarpa Schiede, Pinus caribaea var. hondurensis Barr. \& Golf, e Pinus maximinoi H. E. Moore e métodos de seleção para melhoramento genético. 1996. 169 p. Tese (Doutorado em Ciências Florestais) - Universidade Federal do Paraná, Curitiba, 1996.

STURION, J. A. Variação genética de características de crescimento e de qualidade da madeira em progênies de Eucalyptus viminalis LABILL. 1993. 112 p. Tese (Doutorado em Ciências Florestais) - Setor de Ciências Agrárias, Universidade Federal do Paraná, Curitiba, 1993.

Recebido em 05/05/2016

Aceito para publicação em 17/01/2017 